\title{
On the torsion effects of a relativistic spin fluid in early cosmology
}

\author{
G. de Berredo-Peixoto ${ }^{1 *}$ and E.A. de Freitas ${ }^{1,2} \dagger$ \\ 1. Departamento de Física, ICE, Universidade Federal de Juiz de Fora \\ Campus Universitário - Juiz de Fora, MG Brazil 36036-330 \\ 2. Colégio Técnico Universitário, Universidade Federal de Juiz de Fora \\ Av Bernardo Mascarenhas, 1283 - Juiz de Fora, MG Brazil 36080-001
}

\begin{abstract}
In this work we investigate the effects of torsion in the framework of Einstein-Cartan theory in early cosmology. We study solutions for a homogeneous and isotropic relativistic Weyssenhoff spin fluid with dynamical timelike axial current, also homogeneous and isotropic. The general solutions can mostly be described by means of three particular solutions. The properties of these solutions (such as singularity avoidance and primordial or late accelerated expansion) are analysed and depend on the relations between the source parameters.
\end{abstract}

PACS: $\quad 04.20 .-\mathrm{q} \quad$ 04.50.Kd $\quad 98.80 .-\mathrm{k}$

Keywords: Torsion, Weyssenhoff fluid, Cosmology, Inflation.

\footnotetext{
*Email: gbpeixoto@hotmail.com

${ }^{\dagger}$ Email: emanuel@fisica.ufjf.br
} 


\section{Introduction}

The effects of non-Riemannian structures in the framework of Cosmology have been studied long ago. The most simple generalization of General Relativity (preserving metricity) is achieved by the introduction of an asymetric connection, with torsion as its antisymmetric part. The Riemannian case is obtained by imposing zero torsion. It is possible to consider cosmological models in the framework of more general non-Riemannian structures, such as non-metricity (see for example the review by Puetzfeld [1]), but these cases will not be treated here. There are a large number of papers dealing with torsion in several approaches, with wide applications. For the introduction of the foundations of the theory of gravity with torsion, see Ref. [2], and for a more recent review, including the quantum aspects of torsion, see Ref. [3].

In the seventies, it was discovered that the singularity avoidance and inflation can be induced by torsion in the Einstein-Cartan theory, by Kopczynski [4], Trautman [5] and Hehl et al [6]. In the Einstein-Cartan theory, torsion is not dynamical and is completely expressed in terms of the spin sources [2]. Thus, in order to study the effects of torsion in the Einstein-Cartan theory, one has to introduce matter with spin. This can be done in several ways. One of the most natural ways is to consider, besides the Einstein-Cartan action, the Lagrangian describing spin- $1 / 2$ Dirac fields, minimally interacting with torsion. It is possible to describe this theory as a modified General Relativity with a spin-spin contact interaction. According to Ref. [7], torsion does not prevent the initial singularity, but rather enhances it. In addition, torsion can provide accelerated expansion phase in a metric-torsion theory with matter described by Dirac and Rarita-Schwinger spinors [8]. In this scenario, the authors of Ref. [9] described the transition between early accelerated expansion and decelerated one in terms of massive Dirac fields (see also, for example, Ref. [10]). It is possible of course to consider another Lagrangian for gravity and torsion, in a more general context of Poincaré Gauge Theory of Gravity, with quadratic terms in curvature strenghs. Within this possibility, one can mention for example the works in Refs. [11, 12] (see also references therein), which take into account a spinless matter.

Another way to introduce torsion is to consider a fluid with intrinsic spin density, which in principle does not admit a Lagrangian description based on spinor fields. One has to postulate a spin correction to the energy-momentum tensor. For exemple, Szydlowski and Krawiec [13] have studied the cosmological effects of an exotic perfect fluid ${ }^{1}$ known as the Weyssenhoff fluid [15], as well as the constraints from supernovae Ia type observations, concluding that the dust Weyssenhoff fluid provides accelerated expansion but it can not serve as an alternative to Dark Energy. Also, in Ref. [16], Puetzfeld and Chen derived some constraints from supernovae Ia data in a different scenario of non-Riemannian geometry. Gasperini [17] considered the Weyssenhoff fluid with its energy momentum tensor (derivable from a Lagrangian) previoulsy improved by Ray and Smalley [18], with spin as a thermodynamical variable. In Ref. [17], torsion provides singularity avoidance and early accelerated expansion, but the expansion factor of the cosmological scale, $a(t)$, is too small, unless the state equation parameter $w(p=w \rho)$ of the spin fluid is fine tunned in a very

\footnotetext{
${ }^{1}$ This fluid presents an intrinsic spin density (See also Ref. [14] where a quantum treatment was applied for this fluid in early cosmology).
} 
special way. See also Ref. [19] where similar analysis was performed in a more general context without assuming a particular metric. Obukhov and Korotky [20] formulated a more general variational theory describing the Weyssenhoff fluid and also applied to cosmological models with rotation, shear and expansion. Recently, Böhmer and Burnett [21] introduced a special spinorial matter satisfying the Cosmological Principle [22] (see also [23]), which, together with interaction terms and without cosmological constant, mimics vacuum energy responsible for inflation.

It is worth mentioning that many works without torsion but with different matter sources (besides the scalar field) inducing inflation can be found in literature. For example, Golounev, Mukhanov and Vanchurin [24] proposed a scenario with massive non-minimally coupled vector field, which induces inflation. In homogeneous and isotropic universe, these vector fields behave as a minimally coupled massive field, and they can be introduced as an orthogonal triplet or in a large number randomly oriented in order to provide isotropic inflation. There are no clear understanding about the physical motivations for these vector fields. The authors of Ref. [25] consider also time-like non-minimally coupled vector fields (see also [26] where cosmological perturbations were studied). Among theories without torsion, one can mention also Ref. [27], where the authors show that the dark energy can be described by means of the usual electrodynamics with a non-linear adding term. Also, one can consider a timelike vector field, responsible for violation of Lorentz symmetry [28]. In the present work, the timelike axial current not only violates Lorentz symmetry, but it is related to torsion in the context of the Einstein-Cartan theory, and it is originated by the Weyssenhoff fluid. On the other side, we can cite Ref. [29], where the possibility of inflation induced by non-standard spinors was investigated, as well as their imprints on CMB anisotropies.

We consider the Einstein-Cartan theory with both the spin-spin contact interaction and the Weyssenhoff fluid. In practice, one has an axial current and a spin density as additional sources besides the usual perfect fluid energy density and pressure. In early universe, any matter content has very high temperature, so we let $w$ fixed corresponding to a radiation fluid ( $w=1 / 3)$, which mimics the ultra-relativistic regime. If the model is supposed to describe early inflation, then the spin fluid is an exotic form of matter which can play the role of vacuum energy. The matter content of the model is given by this exotic fluid (with $w=1 / 3$ ) plus a timelike axial current, homogeneous and isotropic. The anisotropic case is very interesting (providing description of primordial anisotropy), although it is beyond the framework of the present work.

This paper is organized as follows. In section 2, we shall present a brief introduction to EinsteinCartan theory, including the dynamical equations of the model. In section 3 , we investigate the solutions of the model for different cases. In section 4, we draw our conclusions and final remarks.

\section{Einstein-Cartan Theory and dynamical equations}

The action in the Einstein-Cartan framework is given by

$$
S=\int \sqrt{-g} d^{4} x\left\{-\frac{1}{\kappa^{2}}(\tilde{R}-2 \Lambda)+\mathcal{L}_{M}\right\},
$$


where metric has signature $\left(+---\right.$ ), $\kappa^{2}=16 \pi G$ (we use units such that $\hbar=c=1$ ), $\Lambda$ is the cosmological constant and $\tilde{R}$ is the Ricci scalar constructed with the asymmetric connection ${ }^{2}, \tilde{\Gamma}^{\mu}{ }_{\alpha \beta}$, which, by using the metricity condition $\left(\tilde{\nabla}_{\alpha} g_{\mu \nu}=0\right)$ and the following definition of torsion,

$$
T_{\alpha \beta}^{\mu}:=\tilde{\Gamma}_{\alpha \beta}^{\mu}-\tilde{\Gamma}_{\beta \alpha}^{\mu},
$$

can be expressed as

$$
\tilde{\Gamma}_{\alpha \beta}^{\mu}=\Gamma_{\alpha \beta}^{\mu}+K_{\alpha \beta}^{\mu},
$$

where $\Gamma^{\mu}{ }_{\alpha \beta}$ is the Riemannian connection (Levi-Civita connection) and the quantity $K^{\mu}{ }_{\alpha \beta}$ is the contortion tensor, given by

$$
K_{\alpha \beta}^{\mu}=\frac{1}{2}\left(T_{\alpha \beta}^{\mu}-T_{\alpha}{ }^{\mu}{ }_{\beta}-T_{\beta}{ }^{\mu}{ }_{\alpha}\right) .
$$

The term $\mathcal{L}_{M}$ is the Lagrangian describing matter distribution. We consider here the following matter Lagrangian:

$$
\mathcal{L}_{M}=\mathcal{L}_{A C}+\mathcal{L}_{S F},
$$

where $\mathcal{L}_{S F}$ is the Lagrangian of the spin fluid [18] and $\mathcal{L}_{A C}$ is the external source, present in the minimally coupling Dirac sector (see, e.g., [3]):

$$
\mathcal{L}_{A C}=J^{\mu} S_{\mu}
$$

Here, $S_{\mu}$ is the axial part of torsion, defined by $S_{\mu}=\varepsilon_{\lambda \rho \sigma \mu} T^{\lambda \rho \sigma}\left(\varepsilon_{\lambda \rho \sigma \mu}\right.$ is the Levi-Civita tensor, with $\varepsilon_{0123}=\sqrt{-g}$ ), and $J^{\mu}$ is the external axial current ${ }^{3}, J^{\mu}=<\bar{\psi} \gamma^{5} \gamma^{\mu} \psi>$, where this average is due to quantum effects (see [3]), such that $J^{\mu}$ is a vacuum property, responsible for Lorentz violation (see also [30]).

In order to vary the action and get the dynamical equations, one has to define what are the independent variables. We choose $g^{\mu \nu}$ and $T^{\alpha}{ }_{\beta \gamma}$ as independent dynamical variables, and $J^{\mu}$ as a quantity defined by the symmetry violation of the vacuum, which is, as we shall see, dependent from dynamical variable $g^{\mu \nu}$. The spacetime metric is the spatially flat homogeneous and isotropic metric such that

$$
d s^{2}=d t^{2}-a(t)^{2}\left(d x^{2}+d y^{2}+d z^{2}\right) .
$$

It is natural to assume that, in comoving frame, $J^{\mu}$ is a homogeneous and isotropic vector, otherwise it would break the isotropy of the universe. Thus, $J^{\mu}$ is a timelike vector so that we let $J^{\mu} J_{\mu}=J^{2}(t)$.

\footnotetext{
${ }^{2}$ All quantities with an upper tilde are constructed with the asymmetric connection, and the corresponding quantities without tilde are constructed with the Riemannian (symmetric) conection.

${ }^{3}$ The matrix $\gamma^{5}$ is the chiral Dirac matrix $\gamma^{5}=(i / 4 !) \varepsilon^{\alpha \beta \mu \nu} \gamma_{\alpha} \gamma_{\beta} \gamma_{\mu} \gamma_{\nu}=i \gamma_{0} \gamma_{1} \gamma_{2} \gamma_{3}$.
} 


\subsection{Variational principle and dynamical equations}

The dynamical equations for metric fields and torsion, in terms of the sources, can be obtained respectively by the usual procedure ${ }^{4}$

$$
\begin{aligned}
\frac{\delta S}{\delta g^{\mu \nu}}=0 & \Longrightarrow \frac{1}{\sqrt{-g}} \frac{\delta(\sqrt{-g} \tilde{R})}{\delta g^{\mu \nu}}=\frac{\kappa^{2}}{2} T_{\mu \nu} \\
\frac{\delta S}{\delta T^{\mu}{ }_{\nu \alpha}}=0 & \Longrightarrow \frac{1}{\sqrt{-g}} \frac{\delta(\sqrt{-g} \tilde{R})}{\delta T^{\mu}{ }_{\nu \alpha}}=\kappa^{2} \theta_{\mu}{ }^{\nu \alpha}
\end{aligned}
$$

where

$$
T_{\mu \nu}:=\frac{2}{\sqrt{-g}} \frac{\delta\left(\sqrt{-g}\left(\mathcal{L}_{A C}+\mathcal{L}_{S F}\right)\right)}{\delta g^{\mu \nu}}:=T_{\mu \nu}^{A C}+T_{\mu \nu}^{S F}
$$

and

$$
\theta_{\mu}{ }^{\nu \alpha}:=\frac{1}{\sqrt{-g}} \frac{\delta\left(\sqrt{-g}\left(\mathcal{L}_{A C}+\mathcal{L}_{S F}\right)\right)}{\delta T^{\mu}{ }_{\nu \alpha}}:=\left(\theta_{A C}\right)_{\mu}{ }^{\nu \alpha}+\left(\theta_{S F}\right)_{\mu}{ }^{\nu \alpha} .
$$

It should be noticed that instead of procedure (7), one can verify that

$$
\frac{\delta S}{\delta K_{\mu \nu \alpha}}=0 \text { gives } \frac{1}{\sqrt{-g}} \frac{\delta(\sqrt{-g} \tilde{R})}{\delta K_{\mu \nu \alpha}}=\kappa^{2} \tau^{\mu \nu \alpha},
$$

where

$$
\tau^{\mu \nu \alpha}:=\frac{1}{\sqrt{-g}} \frac{\delta\left(\sqrt{-g} \mathcal{L}_{M}\right)}{\delta K_{\mu \nu \alpha}}:=\tau_{A C}^{\mu \nu \alpha}+\tau_{S F}^{\mu \nu \alpha} .
$$

Equation (8) is totally equivalent to equation (7), thus we shall use it for the convenient correspondence with notations in literature.

By the convention for the curvature tensor in the form ${ }^{5} \tilde{R}_{\lambda \alpha \beta}^{\mu}=2 \tilde{\Gamma}_{\lambda[\beta, \alpha]}^{\mu}+\tilde{\Gamma}_{\lambda \beta}^{\rho} \tilde{\Gamma}_{\rho \alpha}^{\mu}-$ $\tilde{\Gamma}_{\lambda \alpha}^{\rho} \tilde{\Gamma}_{\rho \beta}^{\mu}$, one can achieve, disregarding total derivatives, the relation

$$
\int d^{4} x \sqrt{-g} \tilde{R}=\int d^{4} x \sqrt{-g}\left(R+K_{\rho \alpha}^{\alpha} K_{\lambda}^{\rho \lambda}-K_{\rho \lambda}^{\alpha} K_{\alpha}^{\rho \lambda}\right)
$$

Variation with respect to $T^{\mu}{ }_{\alpha \beta}$ gives

$$
T^{\mu \alpha \beta}+2 g^{\mu[\alpha} T^{\beta]}=\kappa^{2} \tau^{\beta \alpha \mu},
$$

where $T^{\beta}=T^{\rho \beta}{ }_{\rho}$. In order to express $\tau^{\beta \alpha \mu}$, let us mention that the contribution from spin fluid is given by $\tau_{S F}^{\beta \alpha \mu}=-\frac{1}{2} S^{\beta \alpha} u^{\mu}$ [18], where $S^{\beta \alpha}$ is the spin tensor (antissymmetric), and $u^{\mu}$ is the fluid four-velocity ${ }^{6}$. By straightforward algebra, we can obtain the expression for $\tau^{\beta \alpha \mu}=\tau_{A C}^{\beta \alpha \mu}+\tau_{S F}^{\beta \alpha \mu}$ and consequently,

$$
T^{\mu \alpha \beta}+2 g^{\mu[\alpha} T^{\beta]}=\kappa^{2}\left\{-\frac{1}{2} S^{\beta \alpha} u^{\mu}+2 \varepsilon^{\beta \alpha \mu \rho} J_{\rho}\right\} .
$$

\footnotetext{
${ }^{4}$ We use similar notations from literature, e.g., Ref. [17].

${ }^{5}$ We use the symbols [] and ( ) to denote antisymmetrization and symmetrization, according to

$$
A_{[\mu \nu]}=\frac{1}{2}\left(A_{\mu \nu}-A_{\nu \mu}\right) \quad \text { and } \quad A_{(\mu \nu)}=\frac{1}{2}\left(A_{\mu \nu}+A_{\nu \mu}\right)
$$

${ }^{6}$ In previous papers, like, e.g., Ref. [17], the expression $\tau_{S F}^{\beta \alpha \mu}$ has the opposite sign. The reason is that we adopt $L_{S F}$ with different sign in order to reproduce the same fluid dynamical equations usual in literature.
} 
Using the Weyssenhoff condition ${ }^{7}, S^{\beta \alpha} u_{\alpha}=0$, from the above equation we can derive

$$
T^{\mu \alpha \beta}=-\kappa^{2}\left\{2 \varepsilon^{\mu \alpha \beta \rho} J_{\rho}-\frac{1}{2} S^{\alpha \beta} u^{\mu}\right\}
$$

and

$$
S_{\sigma}=\varepsilon_{\mu \alpha \beta \sigma}\left(2 K^{\mu \alpha \beta}\right)=12 \kappa^{2} J_{\sigma}+\frac{1}{2} \kappa^{2} \varepsilon_{\mu \alpha \beta \sigma} S^{\mu \alpha} u^{\beta} .
$$

Variation with respect to $g^{\mu \nu}$ can be done in a straightforward way, giving

$$
\begin{aligned}
G_{\mu \nu}-g_{\mu \nu} \Lambda & -K_{\mu \alpha}^{\alpha} K_{\nu \lambda}^{\lambda}-\frac{1}{2} T_{\rho \mu}^{\alpha} T_{\nu \alpha}^{\rho}-\frac{1}{2} T_{\mu \lambda}^{\alpha} T_{\alpha}{ }^{\lambda}{ }_{\nu}-\frac{1}{4} T_{\mu \rho \lambda} T_{\nu}{ }^{\rho \lambda} \\
& +\frac{1}{8} g_{\mu \nu}\left(4 K^{\alpha}{ }_{\rho \alpha} K^{\lambda \rho}{ }_{\lambda}+2 T^{\alpha \rho \lambda} T_{\rho \lambda \alpha}-T^{\alpha \rho \lambda} T_{\alpha \rho \lambda}\right)=\frac{\kappa^{2}}{2} T_{\mu \nu} .
\end{aligned}
$$

To write the dynamical equations, one can substitute (11) and (12) into the appropriate quantities in (13), including $T_{\mu \nu}$. In this way we can rewrite equation (13) in the form

$$
\begin{aligned}
G_{\mu \nu}-g_{\mu \nu} \Lambda & -\kappa^{4}\left\{g_{\mu \nu} J^{2}+2 J_{\mu} J_{\nu}-\frac{1}{4} g_{\mu \nu} J_{\sigma} \varepsilon^{\alpha \rho \lambda \sigma} S_{\rho \lambda} u_{\alpha}-\frac{1}{2} J_{\sigma} \varepsilon_{(\mu}{ }^{\rho \lambda \sigma} u_{\nu)} S_{\rho \lambda}\right. \\
& \left.+\frac{1}{32} g_{\mu \nu} S_{\rho \lambda} S^{\rho \lambda}-\frac{1}{8} S_{\mu \lambda} S_{\nu}{ }^{\lambda}+\frac{1}{16} u_{\mu} u_{\nu} S_{\rho \lambda} S^{\rho \lambda}\right\}=\frac{\kappa^{2}}{2}\left(T_{\mu \nu}^{A C}+T_{\mu \nu}^{S F}\right)
\end{aligned}
$$

where $J^{2}=J_{\sigma} J^{\sigma}$. Notice that from (11) one has $T^{\alpha}{ }_{\mu \alpha}=0$, such that several terms in (13) vanish. Thus, with axial current and the spin fluid satisfying the Frenkel condition, $S^{\beta \alpha} u_{\alpha}=0$, there are only traceless degrees of freedom of torsion.

The next step is averaging the above equation. For this purpose, a natural and simple assumption is $\left\langle S_{\alpha \beta}\right\rangle=0$, which means that although the spin tensor might have a particular direction in the microscopic scale, its mean value vanishes at macroscopic domain (i.e., the particles have a randomic spin distribution). Let us define (see Ref. [17])

$$
<S_{\alpha \beta} S^{\alpha \beta}>=2 \sigma^{2},
$$

such that

$$
<S_{\mu}^{\lambda} S_{\nu \lambda}>=\frac{2}{3}\left(g_{\mu \nu}-u_{\mu} u_{\nu}\right) \sigma^{2} .
$$

Now we have to express $T_{\mu \nu}^{A C}$ and $T_{\mu \nu}^{S F}$ in terms of the sources. For $T_{\mu \nu}^{S F}$, one can obtain the formula (see, e.g., Ref. [17]):

$$
\begin{aligned}
T_{\alpha \beta}^{S F} & =u_{(\alpha} S_{\beta)}{ }^{\mu} u^{\nu} K^{\rho}{ }_{\mu \nu} u_{\rho}+u^{\rho} K^{\mu}{ }_{\sigma \rho} u^{\sigma} u_{(\alpha} S_{\beta) \mu}-\frac{1}{2} u_{(\alpha} T_{\beta) \mu \nu} S^{\mu \nu} \\
& +\frac{1}{2} T_{\nu \mu(\alpha} S^{\mu}{ }_{\beta)} u^{\nu}+2 \kappa^{2}\left\{(\rho+p) u_{\alpha} u_{\beta}-p g_{\alpha \beta}\right\} .
\end{aligned}
$$

Substituting (11) into (16), one obtains

$$
\begin{aligned}
T_{\alpha \beta}^{S F} & =\kappa^{2} J^{\rho} u_{(\alpha} \varepsilon_{\beta) \mu \nu \rho} S^{\mu \nu}-\kappa^{2} J^{\rho} S^{\mu}{ }_{(\beta} \varepsilon_{\alpha) \mu \nu \rho} u^{\nu}-\frac{\kappa^{2}}{4} u_{\alpha} u_{\beta} S_{\mu \nu} S^{\mu \nu}+\frac{\kappa^{2}}{4} S_{\mu \alpha} S^{\mu}{ }_{\beta} \\
& +2 \kappa^{2}\left\{(\rho+p) u_{\alpha} u_{\beta}-p g_{\alpha \beta}\right\} .
\end{aligned}
$$

\footnotetext{
${ }^{7}$ Also known as the Frenkel condition. It is included by hand, but emerges automatically in the formalism proposed by Obukhov and Korotky [20].
} 
We achieve, by averaging,

$$
\begin{aligned}
<T_{\alpha \beta}^{S F}> & =\kappa^{2}<J^{\rho} u_{(\alpha} \varepsilon_{\beta) \mu \nu \rho} S^{\mu \nu}>-\kappa^{2}<J^{\rho} S^{\mu}{ }_{(\beta} \varepsilon_{\alpha) \mu \nu \rho} u^{\nu}> \\
& -\frac{2 \kappa^{2}}{3} u_{\alpha} u_{\beta} \sigma^{2}+\frac{\kappa^{2}}{6} g_{\alpha \beta} \sigma^{2}+2 \kappa^{2}\left\{(\rho+p) u_{\alpha} u_{\beta}-p g_{\alpha \beta}\right\} .
\end{aligned}
$$

It should be mentioned that, although $\left\langle S_{\mu \nu}>\right.$ vanishes, it is possible to take $<S_{\mu \nu} J^{\alpha}>\neq 0$. Nevertheless, we argue that the assumption $\left\langle S_{\mu \nu} J^{\alpha}>=0\right.$ is correct because $S_{\mu \nu}$ is randomic only in the 3 -space, and $J^{\mu}$ has not any spatial component.

Let us finally express $T_{\mu \nu}^{A C}$ in terms of the sources. The variation of $\sqrt{-g} \mathcal{L}_{A C}$ with respect to $g^{\mu \nu}$ should be done with special care, since $J^{\mu}$ (and also $J_{\mu}$ ) depends on $g^{\mu \nu}$. This computation is done in the Appendix. The result can be written as

$$
T_{\mu \nu}^{A C}=-2 \varepsilon_{(\mu}^{\alpha \beta \rho} T_{\nu) \alpha \beta} J_{\rho}-S_{(\mu} J_{\nu)}
$$

Substituting the sources (11) and (12), we have

$$
T_{\mu \nu}^{A C}=-8 \kappa^{2} g_{\mu \nu} J^{2}-4 \kappa^{2} J_{\mu} J_{\nu}-\kappa^{2} \varepsilon_{(\mu}^{\beta \rho \lambda} u_{\nu)} S_{\beta \rho} J_{\lambda}+\frac{\kappa^{2}}{2} \varepsilon_{(\mu}^{\beta \rho \lambda} J_{\nu)} S_{\rho \lambda} u_{\beta} .
$$

Now, one can obtain the following dynamical equation after averaging (using $<S_{\mu \nu} J^{\alpha}>=0$ ):

$$
G_{\mu \nu}=\kappa^{4}\left\{-3 g_{\mu \nu} J^{2}+\frac{1}{16} g_{\mu \nu} \sigma^{2}-\frac{1}{8} u_{\mu} u_{\nu} \sigma^{2}\right\}+\frac{\kappa^{2}}{2}\left\{(\rho+p) u_{\mu} u_{\nu}-p g_{\mu \nu}\right\}+\Lambda g_{\mu \nu}
$$

\section{Dynamical equations}

Let us consider the fluid in a relativistic regime, such that $p=\rho / 3$. For the metric (5), the relevant components of equation (21) can be written as

$$
\begin{aligned}
\frac{3 \dot{a}^{2}}{a^{2}} & =\kappa^{4}\left\{-3 J^{2}-\frac{\sigma^{2}}{16}\right\}+\frac{\kappa^{2}}{2} \rho+\Lambda \\
-\frac{\dot{a}^{2}}{a^{2}}-\frac{2 \ddot{a}}{a} & =\kappa^{4}\left\{3 J^{2}-\frac{\sigma^{2}}{16}\right\}+\frac{\kappa^{2}}{6} \rho-\Lambda,
\end{aligned}
$$

where the dot above variables means time derivative. From these equations, one can get

$$
\frac{\ddot{a}}{a}=\kappa^{4}\left\{-J^{2}+\frac{\sigma^{2}}{24}\right\}-\frac{\kappa^{2}}{6} \rho+\Lambda / 3 .
$$

It is remarkable that the axial current enters in the above equation with different sign from the spin contribution. In this equation, the axial current acts as a kind of density energy of some ordinary matter. We see that only the cosmological constant and the spin contribute to the accelerated expansion.

The energy conservation law can be obtained by comparing equation (23) with the time derivative of equation (22):

$$
\dot{\rho} a+4 \dot{a} \rho=\kappa^{2}\left\{\frac{1}{8 a^{5}} \frac{d}{d t}\left(a^{6} \sigma^{2}\right)+6 a \frac{d}{d t}\left(J^{2}\right)\right\} .
$$


Let us remark that the above equation describes energy conservation in the general case when all fields interact with each other. Of course, the spin contribution $\left(\sigma^{2}\right)$ always interacts with the fluid itself, because spin is a fluid property. On the other hand, we have freedom to choose an interacting axial current and a non-interacting one. These possibilities define two classes of solutions. The non-interacting axial current case was studied previoulsy in Ref. [31]. The case without spinning fluid can be found in Ref. [3], where a different variational procedure was realized.

Equations (22) and (25), along with the initial conditions $\rho\left(t_{0}\right)=\rho_{0}$ and $a\left(t_{0}\right)=a_{0}=1\left(t_{0}\right.$ is the present time), determine the dynamical solution of the model. For the CMB, we know that $\rho_{0} \sim \rho_{c} \Omega_{\mathrm{rad}}$. As

$$
\Omega_{\mathrm{rad}} \sim 10^{-5} \text { and } \rho_{c}=\frac{3 H^{2}}{8 \pi G} \sim 4 \times 10^{-47}(G e V)^{4}\left(\text { with } H=\frac{\dot{a}}{a}\right),
$$

one can estimate $\rho_{0} \sim 10^{-52}(\mathrm{GeV})^{4}$ for pure radiation. Of course, this value does not necessarily correspond to the exotic spin fluid, but can serve as a reference (or upper bound). In what follows, we shall consider in most cases a much lower density for the spinning fluid, say, $\rho_{0} \sim 10^{-54}(G e V)^{4}$.

\section{Solutions with interacting axial current}

In searching for solutions, we should specify the dependence of $J^{2}$ and $\sigma^{2}$ on the density, $\rho$. One can adopt $\sigma^{2}=\gamma \rho^{3 / 2}(\gamma=$ positive constant) [32]. We identify the axial current as coming from the spinning fluid. In doing so, the natural choice for $J^{2}$ is $J^{2}=\beta \rho^{3 / 2}$ ( $\beta=$ positive constant). With these assumptions, the dynamical equations for the model are

$$
\dot{\rho}=\frac{12 \kappa^{2} \dot{a} \gamma \rho^{3 / 2}-64 \dot{a} \rho}{16 a-3 \kappa^{2} a \gamma \rho^{1 / 2}-144 \kappa^{2} a \beta \rho^{1 / 2}}
$$

and

$$
\dot{a}=a \sqrt{\frac{\kappa^{2}}{6} \rho+\frac{\Lambda}{3}-\kappa^{4} \rho^{3 / 2}\left(\beta+\frac{1}{48} \gamma\right)} .
$$

The above system is very complicated and can not be solved analytically. However, we can extract relevant information just from (26). Let us rewrite it:

$$
\frac{d \rho}{d a}=\frac{12 \kappa^{2} \gamma \rho^{3 / 2}-64 \rho}{16 a-3 \kappa^{2} a \gamma \rho^{1 / 2}-144 \kappa^{2} a \beta \rho^{1 / 2}} .
$$

For a particular $\rho=\rho_{f}$, there is a fixed point, i.e., $d \rho / d a=0$. When $\rho(a)$ reaches $\rho_{f}$, it ceases to vary. One can express $\rho_{f}$ as

$$
\rho_{f}=\frac{256}{9 \kappa^{4} \gamma^{2}} .
$$

Also, there is an apparent singularity when $\rho=\rho_{c}$ such that $d \rho / d a \rightarrow \infty$. The value for $\rho_{c}$ is obtained by straighforward algebra:

$$
\rho_{c}=\frac{256}{\left(3 \kappa^{2} \gamma+144 \kappa^{2} \beta\right)^{2}}
$$


Actually, the system is not only apparently singular at $\rho_{c}$, but it is really singular, because $\dot{a}$ (from (27)) can not vanish for $\rho_{c}$. Notice that always $\rho_{c}<\rho_{f}$. Let us investigate the three unique possibilities: (i) $\rho_{0}<\rho_{c}<\rho_{f}$; (ii) $\rho_{c}<\rho_{0} \leq \rho_{f}$ and (iii) $\rho_{c}<\rho_{f} \leq \rho_{0}$. For the first possibility, we have $\rho^{\prime}<0$ in the whole interval $\rho<\rho_{c}$. Thus, as time goes backward, density is increased until it reaches $\rho_{c}$. It means that in some finite $a>0$ in the past, $\rho^{\prime}$ is infinite. As it has not clear meaning, we must reject the possibility (i).

For the second possibility, we have $\rho^{\prime}>0$ in the whole interval $\rho_{c}<\rho<\rho_{f}$. Using similar reasoning as before, $\rho^{\prime}$ becomes again infinite for some finite $a$ in the past. In the last possibility, $\rho^{\prime}<0$ in the interval $\rho_{f} \leq \rho$, such that $\rho$ never reaches $\rho_{c}$. Thus, the range given under (iii) appears to be the only viable physical choice.

By the above considerations, the following inequality must be satisfied:

$$
\frac{256}{9 \kappa^{4} \gamma^{2}} \leq \rho_{0}
$$

In numbers, it means that the dimensionless parameter $\gamma$ has a lower bound determined by $\rho_{0}$. For $\rho_{0}=10^{-54} \mathrm{GeV}^{4}$, we achieve a very large lower bound ${ }^{8}: \gamma \geq 1.58 \times 10^{64}$ ! This lower bound imposes the lower bound $\sigma^{2}\left(t_{0}\right)=\gamma \rho_{0}^{3 / 2} \geq 1.6 \times 10^{-17} \mathrm{GeV}^{6}$.

However, a carefull analysis shows that the assumption $\sigma^{2}, J^{2} \propto \rho^{3 / 2}$ is not rigorous. Indeed, one should start from a more simple and fundamental assumption, by considering the dependence of $\sigma^{2}$ and $J^{2}$ (or $\left.(\bar{\psi} \psi)^{2}\right)$ on the scale factor, as

$$
\sigma^{2}, J^{2} \propto a^{-6} .
$$

Notice that in the previous paper [17] the fluid density satisfies $\rho \propto a^{-4}$, such that $\sigma^{2} \propto \rho^{3 / 2}$. Nevertheless, $\rho \propto a^{-4}$ is clearly not an exact solution of the equation (25). Thus, the ansatz (29) will be adopted from now on. Let then

$$
J^{2}=\frac{J_{0}^{2}}{a^{6}} \quad \text { and } \quad \sigma^{2}=\frac{\sigma_{0}^{2}}{a^{6}} .
$$

The equations of motion (22) and (25) can be written in the form

$$
\begin{gathered}
\frac{\dot{a}^{2}}{a^{2}}=-\kappa^{4}\left(\frac{J_{0}^{2}}{a^{6}}+\frac{\sigma_{0}^{2}}{48 a^{6}}\right)+\frac{\kappa^{2}}{6} \rho+\frac{\Lambda}{3}, \\
\frac{d \rho}{d a}=-\frac{4 \rho}{a}-\frac{36 \kappa^{2} J_{0}^{2}}{a^{7}} .
\end{gathered}
$$

It is quite difficult to solve analytically the above system, so it is convenient to treat it numerically. The first observation is that the quantity $d \rho / d a$ in equation (32) is negative and lower than $-4 \rho / a$, thus the effect of the axial torsion is that, as far as $\rho_{0}\left(=\rho\left(t_{0}\right)\right)$ is taken to be the same quantity for both cases (with and without axial current), the values for $\rho$ are higher for $t<t_{0}$.

Now, let us consider equation (31). Its right hand side must be positive for all values of $a(t)$. Choosing the special case $a=1$, we arrive at the following condition (for $\rho_{0}=10^{-54} \mathrm{GeV}^{4}$ ):

$$
J_{0}^{2}+\frac{\sigma_{0}^{2}}{48}<\frac{\rho_{0}}{6 \kappa^{2}}+\frac{\Lambda}{3 \kappa^{4}} \approx 4.38 \times 10^{-11} \mathrm{GeV}^{6}
$$

\footnotetext{
${ }^{8}$ Notice that $\Lambda=5 \times 10^{-84} \mathrm{GeV}^{2}$ and $\kappa^{2}=3.38 \times 10^{-37} \mathrm{GeV}^{-2}$.
} 
which defines an upper bound constraint for the source parameters $J_{0}^{2}$ and $\sigma_{0}^{2}$. In equation (31), the term proportional to $\kappa^{4}$ has the same dependence on the scale factor than the non-Riemannian parameters in Ref. [16]. Although a different model is studied in this work, this coincidence opens the possibility for estimating some constraints from supernovae Ia data applied to the present model ${ }^{9}$. As a result, we get $J_{0}^{2}+\frac{\sigma_{0}^{2}}{48} \leq 5.91 \times 10^{-12} \mathrm{GeV}^{6}$, which is remarkably similar with (33).

Before investigating general solutions for (31) and (32), let us consider the obvious particular solution of (32):

$$
\rho(a)=\frac{\rho_{0}}{a^{6}} .
$$

Substituting this solution into (32), one achieves the constraint

$$
\rho_{0}=18 \kappa^{2} J_{0}^{2} .
$$

Notice that in this case the equation (33) establishes effectively an upper bound for $\sigma_{0}^{2}$, since $J_{0}^{2}$ is attached to $\rho_{0}$. The equation $(31)$ reads

$$
\frac{\dot{a}^{2}}{a^{2}}=\frac{\theta}{a^{6}}+\frac{\Lambda}{3} \quad \text { where } \quad \theta:=\kappa^{4}\left(2 J_{0}^{2}-\frac{\sigma_{0}^{2}}{48}\right) .
$$

There are three solutions depending on the sign of $\theta$ :

$$
a(t)=\left\{\sqrt{\frac{3 \theta}{\Lambda}} \sinh (\sqrt{3 \Lambda} t)\right\}^{1 / 3} \quad \text { for } \theta>0,
$$

where the integration constant was already fixed by $a(0)=0$, and the second solution is given in the implicit form:

$$
a(t)^{3}+\sqrt{\frac{3 \theta}{\Lambda}+a(t)^{6}}=\sqrt{-\frac{3 \theta}{\Lambda}} \exp (\sqrt{3 \Lambda} t) \text { for } \theta<0 .
$$

In the above solution, the integration constant was choosen such that $a(0)=a_{\min }=(-3 \theta / \Lambda)^{1 / 6}$, with $a_{\text {min }}$ being the minimum value of $a$ which can be found by the condition $\theta+\Lambda a^{6} \geq 0$. Thus, for $\theta<0$, there is a singularity avoidance, and the universe undergoes an accelerated expansion all the time. In contrast, solution (37) does not prevent the model from having initial singularity.

The third solution comes from the case $\theta=0$. It is given by $a(t) \propto e^{\sqrt{\Lambda / 3} t}$ (De Sitter). It is remarkable that in this case the Universe expands as it was empty with only the cosmological constant, but there is a fluid with $\rho \propto a^{-6}$.

It is interesting to extract information about the source parameters, $J_{0}^{2}$ and $\sigma_{0}^{2}$, just from the experimental constraints such as the known age of the universe $^{10}, t_{0}=13.7$ billion years $=6.56 \times 10^{41} \mathrm{GeV}^{-1}$. Let us consider, for example, the solution (37). By using the expression for $a(t)$ in (37) at the equality $a\left(t_{0}\right)=1$, one can get $\theta=4.18 \times 10^{-86} \mathrm{GeV}^{2}$ (similar quantity can be found for $|\theta|$ in the case (38)). Now, using equation (24) in the form

$$
\frac{\ddot{a}}{a}=-\frac{2 \theta}{a^{6}}+\frac{\Lambda}{3},
$$

\footnotetext{
${ }^{9}$ We thank the anonymous referee for this indication.

${ }^{10}$ Notice that this proceedure is not rigorous if we remember that in late times, the role of torsion will be supressed by the conventional matter content, such as perfect fluid in dust form.
} 
we achieve, by direct substitution,

$$
\frac{\ddot{a}}{a}=\frac{\Lambda}{3}\left\{1-\operatorname{csch}^{2}(\sqrt{3 \Lambda} t)\right\} .
$$

It is possible to show that this quantity is positive if $t>2.96 \times 10^{41} \mathrm{GeV}^{-1}$, what means that, taking $\theta=4.18 \times 10^{-86} \mathrm{GeV}^{2}$, the expansion of the universe is accelerated for $a>0.61$.

Notice that in the absence of the cosmological constant, the quantity $\theta$ must be positive, admitting then the solution (37). Only a non-null cosmological constant can provide singularity avoidance (for $J_{0}^{2} \neq 0$ ). It is remarkable that the theory with axial current and spin fluid, satisfying (35), requires the introduction of $\Lambda$, otherwise the model would have no solutions for all possible values of $\sigma_{0}^{2}$. For $J_{0}^{2}=0$, the model reduces to the one studied by Gasperini [17], with early and late accelerated expansion, and also singularity avoidance. In this case, the early accelerated expansion takes place in a very short period of time, and it should be mentioned that $J_{0}^{2} \neq 0$ can be taken as an important generalization, in a Lorentz violating theories or as a vacuum quantum effect, for example.

In fact, condition (35) seems to be quite particular. However, as we shall see below, the general solutions for $\rho\left(t_{0}\right) \neq 18 \kappa^{2} J_{0}^{2}$ can be described by the particular solutions dictated by $\rho \propto a^{-6}$.

\section{General Solutions}

According to previous considerations, we know that $\rho \propto a^{-6}$ is a particular solution, which demands (35). Indeed, one can perform numerical integration of equation (32), using the Mathematica software, starting from the point $\rho\left(a_{0}=1\right)=\rho_{0}$ satisfying (35). The integrated curve for $\rho(a)$ will be exactly the curve for $\rho \propto a^{-6}$, which can be drawn as a straigh line with negative slope $(-6)$ in the logarithm scaling.
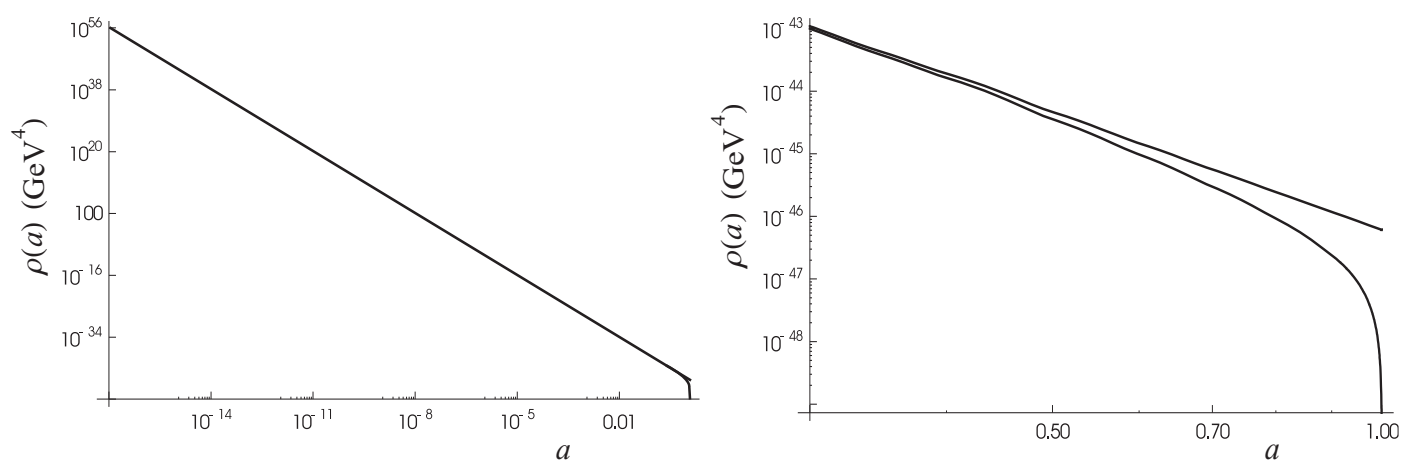

Figure 1: The integral curves, in logarithm scale, for $\rho(a)$, from initial conditions $\rho(1)=10^{-54}$ $\mathrm{GeV}^{4}$ and $\rho(1)=18 \kappa^{2} J_{0}^{2}=6.08 \times 10^{-47} \mathrm{GeV}^{4}$ coincide, except in the small region shown in the right plot.

In order to numerically integrate, we choose some $J_{0}^{2}$ compatible with condition (33), say, $J_{0}^{2}=10^{-11} \mathrm{GeV}^{6}$, and $\rho_{0}$ to be different from (35). Let us choose a lower value: $\rho_{0}=10^{-54} \mathrm{GeV}^{4}$. The integration is shown in the left side of Figure 1, together with the integration obeying (35), corresponding to $\rho \propto a^{-6}$. It is remarkable that both curves coincide, but it is essential to stress 
that they coincide in a certain (and wide) interval. The same plotting in the right side of Figure 1 is drawn in an interval much more closer to $a=1$, where the difference between the two solutions becomes clear.

It seems that this behavior is universal: the curve $\rho(a)$, for any $\rho_{0}$, differs from the solution $\rho \propto a^{-6}$ in some region close to $a=1$, but in the remaining region, say, $0<a<0.1$, the solution is very close to $\rho(a)=18 \kappa^{2} J_{0}^{2} / a^{6}$. Now, remind that there is no much room for big values of $\rho_{0}$, and we are mostly considering early cosmology, thus the particular solutions discussed previously are quite general and instructive.

We should mention that the case $J_{0}^{2}=0[17]$ is completely different, because $\rho(a)$ will be substantially affected, such that $\rho \propto a^{-4}$.

\section{Conclusions and Discussions}

We have investigated the cosmological effects of the axial current together with the relativistic spin fluid $(p=\rho / 3)$ in Einstein-Cartan theory. As already known from literature $[4,5,6]$, torsion provides singularity avoidance and accelerated expansion. The contribution from axial current, however, favours a decelerated expansion, in contrast to the spin fluid.

There are two classes of solutions: one with an external non-interacting axial current, and other with interacting axial current (i.e., time-dependent axial current). The first one was considered in previous works (see [3] for the case without spin fluid and with a conformal global axial vector, and [31] with a global constant vacuum axial vector and a spin fluid).

In the present work, the axial current is assumed as a composite field, $J^{\mu}=<\bar{\psi} \gamma^{5} \gamma^{\mu} \psi>$, where the Dirac fields (presumably) describe the fluid itself. The axial current interacts with the spinning fluid, as realized by the energy-momentum conservation. It is essential that this feature determines the dependence of energy density on the scale factor, $a$, which is substantially different from the case with non-interacting axial current. It is natural to assume that both spin and axial current decay in similar way as the universe expands.

We conclude that, based on the ansatz $J^{2} \propto a^{-6}$ and $\sigma^{2} \propto a^{-6}$, the general solutions behave (in a relevant domain) as the particular solutions coming from $\rho \propto a^{-6}$, such that their properties are the same. These properties were analised in details for the particular solutions: If the source parameter $\theta$ is positive, the universe has an initial singularity and its expansion is decelerated $(\ddot{a}<0)$ until some epoch, when late accelerated expansion begins. This epoch depends basically on the parameter $\theta$, which can be determined from the known age of universe. By doing this, late accelerated expansion starts at $a=0.61$. In both particular solutions, $J_{0}^{2}$ can be fixed by the present value of density, $\rho_{0}$. Thus, $\sigma_{0}^{2}$ determines the sign of $\theta$.

For the case $\theta<0$, universe has an accelerated expansion all the time, and the solution features singularity avoidance. In this case, equation (33) represents an upper bound for the quantities $J_{0}^{2}$ and $\sigma_{0}^{2}$.

As discussed above, the general solutions have a remarkable behavior (as the shift shown in the right side of Figure 1) in the region close to the present day. This is very strange in the physical

point of view, because it would be an enormous coincidence if the shift of the actual curve occurs 
right on the present time, $t_{0}$. Thus, it seems that the physically reasonable solution must be the particular one, $\rho \propto a^{-6}$. As a consequence, the parameter $J_{0}^{2}$ should be related to the present density by equation (35).

\section{Acknowledgments}

The work of the authors has been supported by research grant from CNPq (G.B.P), from FAPEMIG (G.B.P. and E.A.F.) and FAPES (G.B.P.). We would like to express our gratitute to Prof. Ilya Shapiro for stimulating discussions and for relevant suggestions on reading the manuscript.

\section{Appendix: variational proceedure}

Here we shall calculate the functional derivative of $J^{\mu}$. To do so, one must take into account that $J^{\mu}=\bar{\psi} \gamma^{5} \gamma^{\mu} \psi$, with $\gamma^{5}=(i / 4 !) \varepsilon^{\alpha \beta \mu \nu} \gamma_{\alpha} \gamma_{\beta} \gamma_{\mu} \gamma_{\nu}$. As $\gamma_{\mu} \gamma^{\mu}=4$, we can write $\delta\left(\gamma_{\alpha} \gamma^{\alpha}\right) / \delta g^{\mu \nu}=0$. With this equation, one can express $\delta \gamma_{\alpha} / \delta g^{\mu \nu}$ in terms of $\delta \gamma^{\beta} / \delta g^{\mu \nu}$ and vice-versa. Using $\gamma^{\rho}=g^{\rho \lambda} \gamma_{\lambda}$, we achieve

$$
\frac{\delta \gamma^{\rho}}{\delta g^{\mu \nu}}=\frac{1}{2} \delta_{(\mu}^{\rho} \gamma_{\nu)} \quad \text { and } \quad \frac{\delta \gamma_{\rho}}{\delta g^{\mu \nu}}=-\frac{1}{2} g_{\rho(\mu} \gamma_{\nu)}
$$

Now, we know that $\varepsilon^{\alpha \beta \rho \lambda}=E^{\alpha \beta \rho \lambda} / \sqrt{-g}$, where $E^{\alpha \beta \rho \lambda}$ is the Levi-Civita symbol, which is clearly independent on the metric. Thus, we obtain

$$
\frac{\delta \varepsilon^{\alpha \beta \rho \lambda}}{\delta g^{\mu \nu}}=\frac{1}{2} g_{\mu \nu} \varepsilon^{\alpha \beta \rho \lambda} \quad \text { and } \quad \frac{\delta \varepsilon_{\alpha \beta \rho \lambda}}{\delta g^{\mu \nu}}=-\frac{1}{2} g_{\mu \nu} \varepsilon_{\alpha \beta \rho \lambda} .
$$

Using (39) and (40), one can get

$$
\frac{\delta \gamma^{5}}{\delta g^{\mu \nu}}=0
$$

With all these results, one obtains by straighforward algebra the variation of $J^{\alpha}$ and $J_{\alpha}$ :

$$
\frac{\delta J^{\rho}}{\delta g^{\mu \nu}}=\frac{1}{2} \delta_{(\mu}^{\rho} J_{\nu)}, \quad \frac{\delta J_{\rho}}{\delta g^{\mu \nu}}=-\frac{1}{2} \delta_{(\mu}^{\rho} J_{\nu)} \quad \text { and } \quad \frac{\delta J^{2}}{\delta g^{\mu \nu}}=0
$$

Similar computations can be performed for $S^{\lambda}=\varepsilon^{\alpha \beta \rho \lambda} T_{\alpha \beta \rho}=\varepsilon^{\alpha \beta \rho \lambda} g_{\alpha \sigma} T_{\beta \rho}^{\sigma}$. After all, the variation of $\sqrt{-g} J^{\mu} S_{\mu}$ can be expressed by means of result (19).

\section{References}

[1] D. Puetzfeld, New Astron. Rev. 49: 59-64, 2005.

[2] F.W. Hehl, P. von der Heyde, G.D. Kerlick, and J.M. Nester, Rev. Mod. Phys. 48: 393-416, 1976.

[3] I.L. Shapiro, Phys.Rept. 357: 113, 2002.

[4] W. Kopczynski, Phys. Lett. 39A: 219, 1972; 43A: 63, 1973.

[5] A. Trautman, Nature 242: 7, 1973. 
[6] F.W. Hehl, P. von der Heyde and G.D. Kerlick, Phys. Rev D 10: 1066, 1974.

[7] G.D. Kerlick, Phys. Rev. D 12: 3004, 1975.

[8] T. Watanabe and M.J. Hayashi, General Relativity with Torsion, preprint gr-qc/0409029.

[9] M.O. Ribas and G.M. Kremer, Fermion fields in Einstein-Cartan theory and the accelerateddecelerated transition in a primordial Universe, preprint gr-qc/0902.2696.

[10] M.O. Ribas, F.P. Devecchi and G.M. Kremer, Phys. Rev. D 72: 123502, 2005; Europhys. Lett. 81: 19001, 2008.

[11] A.V. Minkevich, A.S. Garkun and V.I. Kudin, Class. Quantum Grav. 24: 5835-5847, 2007.

[12] A.V. Minkevich, Phys. Lett. B678: 423-426, 2009.

[13] M. Szydlowski and A. Krawiec, Phys. Rev. D 70: 043510, 2004.

[14] G.G.A. Bäuerle and C.J. Haneveld, Physica A 121: 541, 1983.

[15] J. Weyssenhoff and A. Raabe, Acta Phys. Pol. 9: 7, 1947.

[16] D. Puetzfeld and X. Chen, Class. Quantum Grav. 21: 2703, 2004.

[17] M. Gasperini, Phys. Rev. Lett. 56: 2873, 1986.

[18] J.R. Ray and L.L. Smalley, Phys. Rev. D 27: 1383, 1983.

[19] S.D. Brechet, M.P. Hobson and A.N. Lasenby, Class. Quantum. Grav. 25: 245016, 2008.

[20] Yu.N. Obukhov and V.A. Korotky, Class. Quantum Grav. 4 1633-1657 (1987).

[21] C.G. Boehmer and J. Burnett, Phys. Rev. D 78: 104001, 2008.

[22] M. Tsamparlis, Phys. Lett. A 75: 27, 1979.

[23] C.G. Boehmer and P. Bronowski, The homogeneous and isotropic Weyssenhoff fluid, preprint gr-qc/0601089.

[24] A. Golounev, V. Mukhanov and V. Vanchurin, JCAP 0806: 009, 2008.

[25] T.S. Koivisto and D.F. Mota, JCAP 0808: 021, 2008.

[26] T.S. Koivisto and D.F. Mota, JCAP 0806: 018, 2008.

[27] M. Novello, S.E.P. Bergliaffa and J. Salim, Phys. Rev. D 69: 127301, 2004.

[28] S.M. Carroll and E.A. Lim, Phys. Rev. D 70: 123525, 2004.

[29] C.G. Boehmer and D.F. Mota, Phys. Lett. B 663: 168-171, 2008.

[30] V.A. Kostelecky , N. Russell and J. Tasson, Phys. Rev. Lett., 100: 111102, 2008. 
[31] G. de Berredo-Peixoto and E.A. de Freitas, Int. Jour. Mod. Phys. A 24: 1652-1655, 2009.

[32] I.S. Nurgaliev and W.N. Ponomariev, Phys. Lett. B 130: 378-379, 1983.

[33] L.P. Chimento and N.A. Zuccala, Astrophys. Space Sci. 254: 37-49, 1997. 